\title{
Isotope effect in dissociative electron attachment to acetylene
}

\author{
O. May, J. Fedor, ${ }^{*}$ and M. Allan \\ Department of Chemistry, University of Fribourg, Chemin du Musée 9, 1700 Fribourg, Switzerland
}

\begin{abstract}
Absolute partial cross sections for dissociative electron attachment to $\mathrm{H}-\mathrm{C} \equiv \mathrm{C}-\mathrm{H}$ and $\mathrm{D}-\mathrm{C} \equiv \mathrm{C}-\mathrm{D}$ have been measured using a time-of-flight ion spectrometer combined with a trochoidal electron monochromator. For $\mathrm{C}_{2} \mathrm{H}_{2}$ the present data are in good agreement with the total cross sections measured previously in our laboratory using the total ion-collection method. The present measurements on deuterated acetylene revealed a pronounced isotope effect at the first dissociative electron attachment band at $3 \mathrm{eV}$, with the cross section for $\mathrm{C}_{2} \mathrm{D}^{-} / \mathrm{C}_{2} \mathrm{D}_{2}$ being 14.4 times smaller than that for $\mathrm{C}_{2} \mathrm{H}^{-} / \mathrm{C}_{2} \mathrm{H}_{2}$. The dominant fragments at the second dissociative attachment band around $8 \mathrm{eV}$ are the light fragments $\mathrm{H}^{-}$or $\mathrm{D}^{-}$. These bands exhibit weaker isotope effects, in agreement with their assignment to Feshbach resonances with narrow autodetachment widths.
\end{abstract}

\section{INTRODUCTION}

Interest in electron-driven chemistry of acetylene $\mathrm{C}_{2} \mathrm{H}_{2}$ is motivated by many areas of research and applications, ranging from studies of upper planetary atmospheres [1] to the use of acetylene-containing plasma for processing in microtechnology and nanotechnology. The relevance of dissociative electron attachment (DEA) in the latter environment is illustrated by the fact that it was found to trigger nucleation of nanoparticles in reactive acetylene plasmas [2]. Quantitative electron impact cross sections are a necessary prerequisite for understanding the plasma environment and are used for modeling of nanoparticle formation in such plasmas [2].

Acetylene has also become a prototype case for theoretical studies of DEA. The recent calculations of Chourou and Orel [3] represent a great advancement in this direction, both because the resonant potential surface, characterized by its energy and geometry-dependent width, was calculated $a b$ initio by the complex Kohn variational method and because three dimensions (the $\mathrm{C} \equiv \mathrm{C}$ and the $\mathrm{C}-\mathrm{H}$ distances and the $\mathrm{CCH}$ angle) were explicitly included in the calculation of the dissociation path. The dissociation was found to involve first bending and then stretching of the $\mathrm{C}-\mathrm{H}$ bond, and can thus not be described by a one-dimensional model generally used to treat DEA. The dissociation channel is in competition with autodetachment in the course of the motion of the nuclear wave packet, and the resulting DEA cross section is thus a very sensitive test of the model used to calculate the resonant potential surface. The calculation of Chourou and Orel [3] was very successful in this respect; their calculated cross section for the production of $\mathrm{C}_{2} \mathrm{H}^{-}$ from $\mathrm{C}_{2} \mathrm{H}_{2}$ at $3 \mathrm{eV}, 3.44 \mathrm{pm}^{2}$, was in an excellent agreement with the experimental value of $3.6 \mathrm{pm}^{2}$ measured previously in our laboratory [4].

A second stringent test of the theory is the isotope effect, where Chourou and Orel [3] predicted that the cross section for deuterated acetylene is $28.9 \times$ smaller than that of normal

\footnotetext{
*Present address: J. Heyrovský Institute of Physical Chemistry, Academy of Sciences of the Czech Republic, Dolejškova 3, Prague 8, Czech Republic
}

acetylene, as a result of an increased importance of the autodetachment channel over dissociation for the slowermoving $\mathrm{D}$ atom. We have not tested this prediction because the ion-collection method used in our previous study was not sensitive enough to measure the small cross section in deuterated acetylene. In this paper we report on the missing cross section, whose measurement was made possible by the improved sensitivity of a recently constructed time-of-flight (TOF) mass filter, which is optimized for quantitative measurements. An additional benefit is that the present cross sections are resolved with respect to mass, an aspect important primarily in the $8 \mathrm{eV}$ region of the spectrum.

Existing DEA studies on acetylene can be briefly summarized as follows. Early experiments by von Trepka and Neuert [5] revealed an intense $\mathrm{C}_{2} \mathrm{H}^{-}$band with an onset at $2.8 \pm 0.2 \mathrm{eV}$, a weaker $\mathrm{C}_{2} \mathrm{H}^{-}$band with an onset at $6.0 \pm 0.3 \mathrm{eV}$, and an intense $\mathrm{C}_{2}^{-}$band together with a weak $\mathrm{H}^{-}$band, both with an onset at $7.6 \pm 0.3 \mathrm{eV}$. Azria and Fiquet-Fayard [6] measured the total absolute dissociative attachment cross sections for the individual bands, using the total ion-collection method, without mass analysis. They also attempted to measure deuterated acetylene but, because of the weak signal intensity, they could only place a lower limit on the isotope effect, a factor of 11. Higher-resolution spectra of the $3 \mathrm{eV} \mathrm{C}_{2} \mathrm{H}^{-}$and the $8 \mathrm{eV} \mathrm{C}_{2}^{-}$bands have been measured by Abouaf et al. [7], who discovered vibrational structure in the $8 \mathrm{eV}$ band. This structure has been confirmed and correlated with the structure observed in the transmission spectrum by Dressler and Allan [8] and Dressler [9].

Independent information on acetylene resonances has been obtained from vibrational excitation $[10,11]$ and electron transmission $[12,8]$ measurements. These methods suggested that the resonance around $3 \mathrm{eV}$ is of ${ }^{2} \Pi_{g}$ symmetry, with a temporary occupation of the $\pi_{g}\left(\pi^{*}\right)$ LUMO of acetylene; the $8 \mathrm{eV}$ band has been assigned to several Feshbach resonances. Gianturco and Stoecklin [13] analyzed the total eigenphase sums in electron-acetylene scattering and report also a weak broad ${ }^{2} \Sigma_{u}$ resonance around $4 \mathrm{eV}$.

\section{EXPERIMENT}

The instrument is based on the total ion-collection apparatus described in our previous publications $[4,14]$. It uses an 


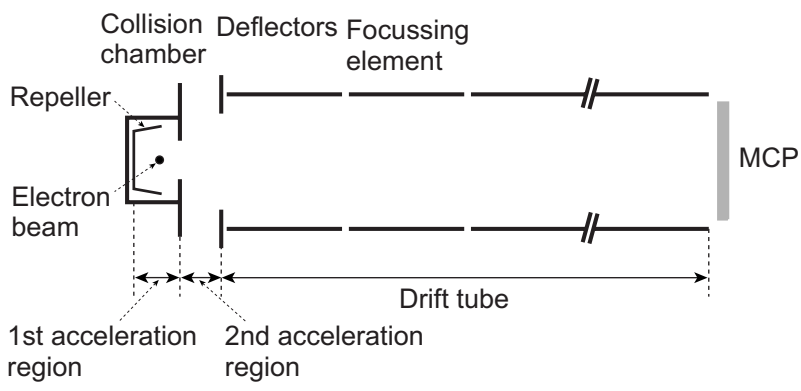

FIG. 1. Scheme of the time-of-flight tube. The orientation of electron beam is perpendicular to the paper plane.

axial magnetic field to collimate the electrons and a trochoidal electron monochromator to reduce the electron energy spread. In the present setup, three of the originally six ion collectors are removed and the corresponding wall of the collision chamber is equipped with an exit slit through which ions enter a TOF tube (Fig. 1). The remaining collectors, on the back wall, are now used as ion repellers. A short (200 ns) pulse of electrons is sent through the interaction region while the ion repellers are on the potential of the chamber. A $4 \mu \mathrm{s}$ long pulse with an amplitude between -300 and $-450 \mathrm{~V}$ is applied to the repellers about $200 \mathrm{~ns}$ later after the electrons have left the collision chamber. The experiment is repeated at a rate of either 10 or $100 \mathrm{kHz}$.

The design of the TOF tube is similar to that of Field and co-workers [15]. The distance between the ion repeller and the ion exit slit (designated as the first accelerating region in Fig. 1) is $12 \mathrm{~mm}$, so that the ions are accelerated over a distance of $6 \mathrm{~mm}$. The second accelerating region and the drift tube are 10 and $140 \mathrm{~mm}$ long, respectively. To minimize the loss of ions and to avoid ion scattering on wire surfaces, we do not use grids to separate individual regions at the cost of a decreased mass resolution.

Measurements of absolute cross sections require that the spectrometer is "quantitative," i.e., that the ion-collection efficiency does not depend on their masses and their initial kinetic energies. To reach this goal, we performed iontrajectory simulations with the program CPO-3D [16] as a guidance and verification during the design. The important issues are that (i) even ions with high initial kinetic energies are extracted from the target chamber, (ii) in particular light ions are not lost because they fly perpendicularly to the magnetic field used to collimate the electron beam, and (iii) even ions that enter the TOF tube with diverging trajectories, as a consequence of their initial kinetic energies, all reach the detector.

Problem (i) is solved by the combination of two design concepts. One is the use of a high repeller voltage, accelerating the ions to energies much higher than their initial kinetic energies. The second concept is the use of an exit slit with a sufficiently large dimension. The critical dimension is that perpendicular to the direction of the electron beam, and it was chosen to be $10 \mathrm{~mm}$. The dimension in the direction of the electron beam is not critical because the ions are sampled from a range of positions along the electron beam, and ions formed in front of the slit and lost because their initial axial velocities are compensated by the same number of ions formed with the same axial velocity at other positions of the beam. This dimension was consequently chosen small, 0.5 $\mathrm{mm}$, to minimize the amount of sample gas escaping through the slit.

The situation is further complicated by the fact that ions formed in the early stages of the electron beam pulse have time to travel a certain distance before the repeller pulse arrives, reducing their chance of being collected. This problem can be reduced, at the expense of signal intensity, by reducing the electron beam pulse duration. We investigated this effect with our own (FORTRAN) code simulating statistical initial ion momenta and times and positions of creation. This and the CPO-3D simulations indicate that the collection efficiency of our design is constant up to an initial ion kinetic energy of $2.5 \mathrm{eV}$, and $90 \%$ remain up to about $4 \mathrm{eV}$.

Problem (ii) is solved by splitting the first section of the TOF tube into two electrostatic $y$ deflectors, used to compensate the effect of the magnetic field. Problem (iii) is solved by designing the TOF tube as a three-cylinder electrostatic lens, which images the ion exit slit onto the multichannel detector. The voltage on the center element, variable up to $V_{2}=2000 \mathrm{~V}$, is used to control the focusing. (The center voltage on the first element and the voltage on the third element are $V_{1}=V_{3}=600 \mathrm{~V}$.) The problem with this design is that the off-axis trajectories are longer, result in a spread of flight times, and reduce the mass resolution. Quantitative ion collection has a priority over the mass resolution in our design, however. The center lens element is also split and is used as a horizontal $(x)$ deflector to steer the ions onto the detector.

The optimum deflecting voltage within the first section of the TOF tube depends on the masses and the initial kinetic energies of the ions. Two sets of settings suffice in practice: one for all "heavy" ions (e.g., $\mathrm{O}^{-}$and $\mathrm{C}_{2} \mathrm{H}^{-}$) and another for the "light" ions $\mathrm{H}^{-}$and $\mathrm{D}^{-}$. Due to momentum conservation, the light ions are often produced with kinetic energies higher than those of the heavy fragments; the divergence of the ions is higher and stronger focusing voltage has to be applied on the drift tube.

The ions that passed the TOF tube are detected with a microchannel plate, counted, and their arrival times are analyzed using the delayed coincidence scheme, with a time-toamplitude converter and a pulse-height analysis (Fast ComTec MCA3-FADC multichannel card). The flight times depend on the voltages on the repeller and in the TOF tube and were around $0.7 \mu \mathrm{s}$ for $\mathrm{H}^{-}$and $2.5 \mu \mathrm{s}$ for $\mathrm{C}_{2}^{-}$. They were converted to a mass scale for presentation in Fig. 2.

An additional potential problem with respect to the requirement of a quantitative mass spectrometer stems from the possible dependence of the detector efficiency on the type of ion. Since we count the ions, the efficiency is given by the quantum yield-the number of signal pulses for a given number of arriving ions. The quantum yield generally depends on the type of ion (its electron affinity) and decreases with increasing mass, a consequence of decreasing velocity of the arriving ions for a given kinetic energy. We attempt to bypass this problem by accelerating the ions to very high energies, where the quantum yields for all ions concerned saturate. Our ion detection system (BURLE BiPolar TOF Detector) uses a scintillator-photomultiplier scheme 


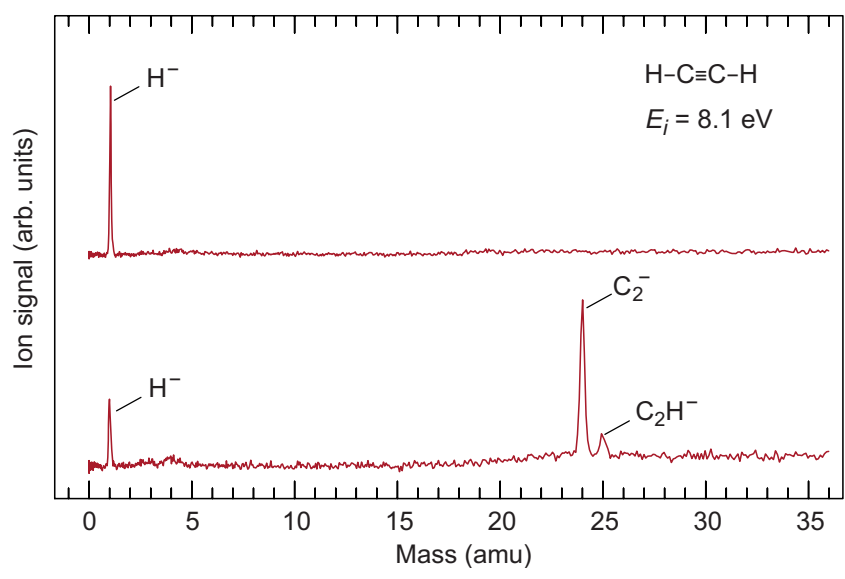

FIG. 2. (Color online) Negative ion TOF mass spectra following DEA to $\mathrm{C}_{2} \mathrm{H}_{2}$ at $8.1 \mathrm{eV}$. Bottom: heavy-ion voltage settings on the time-of-flight tube; top: light-ion settings.

to decouple the very high voltage of the multichannel plate anode from the amplifier input and allows the ion conversion surface of the multichannel plate to be biased up to $+9 \mathrm{kV}$. We have found that the quantum yield of the detector saturates already at $3 \mathrm{kV}$ for all ions investigated $\left(\mathrm{O}^{-}, \mathrm{C}_{2} \mathrm{H}^{-}, \mathrm{C}_{2}^{-}\right.$, and $\mathrm{H}^{-}$) and assume that in this saturation regime the detection efficiency is the same for all of them.

The pressure in the collision chamber is measured by a capacitance manometer and was kept typically between $0.3 \times 10^{-3}$ and $0.6 \times 10^{-3}$ Torr during the measurement. The cross sections were normalized on the $4.4 \mathrm{eV} \mathrm{O}-/ \mathrm{CO}_{2} \mathrm{DEA}$ band. The measurements of this cross section have been reviewed by Orient and Srivastava [17] and by Itikawa [18]. We take a value of $14 \mathrm{pm}^{2}$, which is the average of the values listed by Orient and Srivastava, together with our own value from the total ion-collection experiment. The entire TOF scheme was verified by measuring the cross sections for the $\mathrm{O}^{-} / \mathrm{N}_{2} \mathrm{O}$ and $\mathrm{O}^{-} / \mathrm{O}_{2}$ processes, with the correct results. The energy scale was calibrated on the onset of $\mathrm{O}^{-} / \mathrm{CO}_{2}$ DEA band at $3.99 \mathrm{eV}$. The electron beam current was typically 20-100 nA and the resolution was $200 \mathrm{meV}$. The error of the present relative measurement is $\pm 20 \%$ and has to be combined with the error of the absolute $\mathrm{O}^{-} / \mathrm{CO}_{2}$ cross section used for normalization. The latter is taken as $\pm 15 \%$ in view of the good agreement of the results listed in Ref. [17] and our own result. This results in an error bar (two standard deviations) estimate of $\pm 25 \%$ for the present absolute values.

The thermionic cathode warms the target chamber to about $60{ }^{\circ} \mathrm{C}$ (measured by a Pt100 probe) during the operation. The capacitance manometer is operated at room temperature, about $25^{\circ} \mathrm{C}$, so that the Knudsen correction $p_{h} / p_{l}=\left(T_{h} / T_{l}\right)^{1 / 2}$ (where $p_{h}$ and $p_{l}$ are the pressures at the high and the low temperatures, $T_{h}$ and $T_{l}$, respectively) must be applied to its reading to obtain the correct pressure in the target chamber [19]. This correction is redundant in the present case of time-of-flight measurements, because it applies in the same way to both the measured and the calibrant $\mathrm{CO}_{2}$ gases. On the other hand, the correction should be applied to the absolute measurement using the total ioncollection method. We neglected this correction in our previ-
TABLE I. DEA peak cross sections $\left(\mathrm{pm}^{2}\right)$. The present values are reliable within $\pm 25 \%$.

\begin{tabular}{ccccc}
\hline \hline Target & Energy & Ion & This work & Literature \\
\hline $\mathrm{C}_{2} \mathrm{H}_{2}$ & 3.0 & $\mathrm{C}_{2} \mathrm{H}^{-}$ & 3.45 & $\begin{array}{c}3.8 \pm 0.9[4], 2.2 \pm 0.3[6] \\
3.44[3] \text { (calc.) }\end{array}$ \\
& & & & $4.4 \pm 1[4],{ }^{\mathrm{a}} 3.1 \pm 0.4[6]^{\mathrm{a}}$ \\
& 7.9 & $\mathrm{H}^{-}$ & 3.94 & \\
& 8.3 & $\mathrm{C}_{2}^{-}$ & 0.80 & $\leq 0.19[6]$ \\
$\mathrm{C}_{2} \mathrm{D}_{2}$ & 3.0 & $\mathrm{C}_{2} \mathrm{D}^{-}$ & 0.24 & $0.119[3]$ (calc.) \\
& & & & \\
& 7.7 & $\mathrm{D}^{-}$ & 1.45 & \\
& 8.4 & $\mathrm{C}_{2}^{-}$ & 0.17 & \\
\hline \hline
\end{tabular}

${ }^{a}$ Total cross section measurement; sum of $\mathrm{H}^{-}$and $\mathrm{C}_{2}^{-}$.

ous measurement [4]. Although it is well within the error limit stated as $\pm 25 \%$, we decided to include it into the value quoted in Table I, which is consequently $5.7 \%$ larger than that given in Ref. [4].

\section{RESULTS}

Figure 2 shows the negative ion TOF mass spectra following the dissociative electron attachment to $\mathrm{C}_{2} \mathrm{H}_{2}$ at an electron energy of $8.1 \mathrm{eV}$. The "light-ion settings" provide an increase both in the time resolution and in the collection efficiency for the $\mathrm{H}^{-}$ions. The observed mass resolutions are $m / \Delta m=15$ for $\mathrm{H}^{-}$and $m / \Delta m=80$ at $m=24$, which are sufficient to resolve the peaks corresponding to $\mathrm{C}_{2}^{-}$and $\mathrm{C}_{2} \mathrm{H}^{-}$ fragments.

To obtain the DEA cross sections, we integrate the area under the individual TOF peaks with the background subtracted at each electron energy. Figure 3 shows the resulting DEA spectra of $\mathrm{C}_{2} \mathrm{H}_{2}$ and $\mathrm{C}_{2} \mathrm{D}_{2}$. The measurements were repeated several times and the averages of the peak cross sections are given in Table I. The present cross section value for producing $\mathrm{C}_{2} \mathrm{H}^{-}$from $\mathrm{C}_{2} \mathrm{H}_{2}$ at $3 \mathrm{eV}$ is $3.45 \pm 0.8 \mathrm{pm}^{2}$, in good agreement with our previous measurements in the total ion-collection setup with the result of $3.8 \pm 0.9 \mathrm{pm}^{2}$. (A slightly lower value, $3.6 \pm 0.9 \mathrm{pm}^{2}$, was given for the total ion-collection result in Ref. [4] because of neglect of the Knudsen correction to pressure.) The calculations of Chourou and Orel [3] predict a value of $3.44 \mathrm{pm}^{2}$, which is very close to our experimental values. This agreement is very impressive in view of the fact that the DEA cross section is a very steep function of the primary theoretical result-the autodetachment width of the resonance.

The partial cross sections show that the dominant fragment in the region of the second resonance (around $8 \mathrm{eV}$ ) is $\mathrm{H}^{-}$. In our previous work, with the ion-collection setup without mass analysis, we assigned the band as due primarily to the $\mathrm{C}_{2}^{-}$fragment; this assignment is based on the report of Dressler [9] that the $\mathrm{H}^{-}$signal was very weak. The signal intensity in the experiment of Dressler cannot be judged directly, however, because the weak-field ion extraction scheme used there strongly enhanced very low kinetic energy ions. The initial kinetic energy of the $\mathrm{C}_{2}^{-}$fragment is nearly 

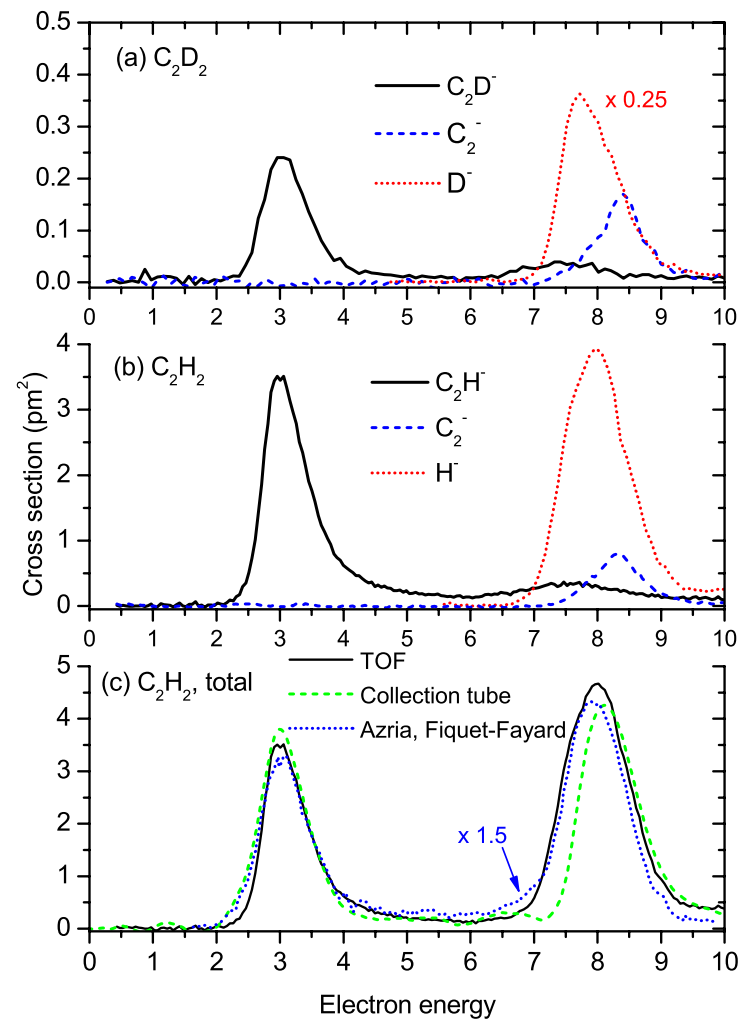

FIG. 3. (Color online) Dissociative electron attachment cross sections for (a) $\mathrm{C}_{2} \mathrm{D}_{2}$ and (b) $\mathrm{C}_{2} \mathrm{H}_{2}$. (c) Total DEA cross section for $\mathrm{C}_{2} \mathrm{H}_{2}$. Full line: sum of partial cross sections from panel (b); dashed line: ion-collection tube data from our laboratory [4]; dots: measurement of Azria and Fiquet-Fayard [6] (scaled to the present values at the $3 \mathrm{eV}$ band).

zero because the neutral fragment $\mathrm{H}_{2}$ is very light, and it was collected efficiently in the experiment of Dressler. On the other hand, Dressler measured a high kinetic energy release, peaking at $1.9 \mathrm{eV}$, for the $\mathrm{H}^{-}$fragment with $7.8 \mathrm{eV}$ incident electrons [9], strongly attenuating the $\mathrm{H}^{-}$signal in his setup. The results of Dressler are thus compatible with the present findings.

Figure 3(c) shows the total DEA cross section for $\mathrm{C}_{2} \mathrm{H}_{2}$ [sum of partial cross sections from Fig. 3(b)] and the total cross section measured previously in our laboratory using the total ion-collection method [4]. Both the absolute cross section and the relative heights of the two peaks are in good agreement. However, the threshold edge of the second peak seems to be shifted considerably toward higher energies in the total ion-collection measurement. For comparison, the total cross section obtained by Azria and Fiquet-Fayard [6] is shown in Fig. 3(c) as well, scaled to our peak cross section of the $3 \mathrm{eV}$ band, to permit an easier comparison of shapes. Their onset of the second band agrees better with the present TOF result. As has been mentioned in Ref. [4], the total ion-collection data were superimposed on the background signal caused by the multiply scattered electrons, which was stronger at higher electron energies. The ambiguity in the background subtraction is probably the cause of the observed difference in the shape of the second band.

We observe a strong isotope effect on the $3 \mathrm{eV}$ band with the ratio $\sigma\left(\mathrm{C}_{2} \mathrm{H}_{2}\right) / \sigma\left(\mathrm{C}_{2} \mathrm{D}_{2}\right)=14.4$ (Table II). Azria and
TABLE II. The isotope effect at the $3 \mathrm{eV}$ band, e.g., the ratio $\sigma\left(\mathrm{C}_{2} \mathrm{H}^{-} / \mathrm{C}_{2} \mathrm{H}_{2}\right) / \sigma\left(\mathrm{C}_{2} \mathrm{D}^{-} / \mathrm{C}_{2} \mathrm{D}_{2}\right)$. The present values are reliable within $\pm 15 \%$.

\begin{tabular}{ccc}
\hline \hline This work & Experiment [6] & Theory [3] \\
\hline 14.4 & $\geq 11$ & 28.9 \\
\hline \hline
\end{tabular}

Fiquet-Fayard [6] gave a lower limit for this ratio to be 11, which is in agreement with our findings. The calculations of Chourou and Orel [3] predicted the ratio of 28.9. Taking into account the extreme sensitivity of the DEA cross sections on the resonance width toward autodetachment and the necessity of treating the nuclear dynamics of $\left(\mathrm{C}_{2} \mathrm{H}_{2}\right)^{-*}$ in three dimensions, the agreement within a factor of 2 with our experimental data can be considered to be good. However, this agreement is worse than the agreement of the $\mathrm{C}_{2} \mathrm{H}^{-} / \mathrm{C}_{2} \mathrm{H}_{2}$ cross section itself. The difference could, at least in part, be due to the dependence of the cross sections on temperature, as will be discussed in more detail below.

The isotope effect in the dissociative channels around 8 $\mathrm{eV}$ is weaker: the cross section ratio is 2.7 in the production of $\mathrm{H}^{-}$or $\mathrm{D}^{-}$and 4.7 for the production of $\mathrm{C}_{2}^{-}$. The $\mathrm{C}_{2}^{-}$bands have vibrational structure when recorded with higher electron energy resolution, revealing a relatively narrow autodetachment width. They have been assigned to several Feshbach resonances with a hole in the $\pi_{u}$ orbital and two electrons in Rydberg-like $3 s$ and/or $3 p$ orbitals [8]. The smaller isotope effect observed for the $8 \mathrm{eV}$ band is compatible with the narrower autodetachment width and thus with the longer lifetime of the resonances deduced from the resolved vibrational structure. The smaller isotope effect is a consequence of a less critical competition of the dissociation and the detachment channels because of the slower autodetachment rate.

\section{CONCLUSIONS}

The present values of the cross sections for electron attachment to $\mathrm{C}_{2} \mathrm{H}_{2}$ agree well with our previous measurements in the total ion-collection tube mode. The capacity of the present experiment to resolve mass confirms that at energies around $3 \mathrm{eV}$ the $\mathrm{C}_{2} \mathrm{H}^{-}$fragment is observed and reveals that the $\mathrm{H}^{-}$fragment dominates above the $\mathrm{C}_{2}^{-}$fragment at the second band at $8 \mathrm{eV}$. The present experiment provides a quantitative value for the isotope effect: the ratio of cross sections for DEA to $\mathrm{C}_{2} \mathrm{H}_{2}$ and $\mathrm{C}_{2} \mathrm{D}_{2}$ is $(14.4 \pm 15) \%$ at $3 \mathrm{eV}$. This value is compatible with the lower limit value of 11 , reported from a total ion-collection experiment by Azria and Fiquet-Fayard [6]. Comparison with the value of 28.9 from the calculation of Chourou and Orel [3] reveals that whereas there is a quantitative agreement with our experimental values for $\mathrm{C}_{2} \mathrm{H}^{-} / \mathrm{C}_{2} \mathrm{H}_{2}$ cross section, the cross section for $\mathrm{C}_{2} \mathrm{D}^{-} / \mathrm{C}_{2} \mathrm{D}_{2}$ is calculated about $2 \times$ lower than the experimental value.

Temperature dependence of the cross section could be, at least in part, responsible for the difference between the measured and the calculated values in the deuterated acetylene. The prototype case of DEA in $\mathrm{H}_{2}$ and $\mathrm{D}_{2}$ shows that in cases 
with a small cross section and a large isotope effect the cross section rises very rapidly with vibrational excitation of the target, and that this rise is much stronger for the deuterated compound, where it reached a factor of 40 for each vibrational quantum [20]. In the same way the DEA cross section in acetylene is very likely to raise dramatically with vibrational excitation of the target, in particular the $\mathrm{CH}$ bending vibrations, and the rise will be much stronger for the deuterated compound. The effect of temperature is further enhanced by the fact that there are two such bending vibrations, $\pi_{g}$ and $\pi_{u}$, with wave numbers of 505 and $537 \mathrm{~cm}^{-1}$ in $\mathrm{C}_{2} \mathrm{D}_{2}$, and each is doubly degenerate, so that their combined population at $333 \mathrm{~K}$ is about $42 \%$, whereas that of the undeuterated acetylene it is only $23 \%$. The rise of the effective cross section (averaged over initial thermal vibrational popu- lation of the target) with temperature, much stronger in $\mathrm{C}_{2} \mathrm{D}_{2}$ than in $\mathrm{C}_{2} \mathrm{H}_{2}$, could thus account, at least in part, for the difference between theory and our experiment. Unfortunately, our present instrument does not allow us to measure the temperature dependence, but a calculation of the cross section for excited initial vibrational levels would be useful. Such a calculation is being worked on by Chourou and Orel [21].

\section{ACKNOWLEDGMENTS}

This research is part of Contract No. 200020-113599/1 of the Swiss National Science Foundation and of COST Action CM0601.
[1] D. E. Shemansky, A. I. F. Stewart, R. A. West, L. W. Esposito, J. T. Hallett, and X. Liu, Science 308, 978 (2005).

[2] M. Mao, J. Benedikt, A. Consoli, and A. Bogaerts, J. Phys. D 41, 225201 (2008).

[3] S. T. Chourou and A. E. Orel, Phys. Rev. A 77, 042709 (2008).

[4] O. May, J. Fedor, B. C. Ibanescu, and M. Allan, Phys. Rev. A 77, 040701(R) (2008).

[5] L. von Trepka and H. Neuert, Z. Naturforsch. A 18A, 1295 (1963).

[6] R. Azria and F. Fiquet-Fayard, J. Phys. (France) 33, 663 (1972).

[7] R. Abouaf, L. Andrić, R. Azria, and M. Tronc, in Proceedings of the 12th International Conference on the Physics of Electronic and Atomic Collisions, edited by S. Datz (NorthHolland, Amsterdam, 1981), p. 409.

[8] R. Dressler and M. Allan, J. Chem. Phys. 87, 4510 (1987).

[9] R. Dressler, Ph.D. thesis, University of Fribourg, 1985.

[10] L. Andrić and R. I. Hall, J. Phys. B 21, 355 (1988).
[11] K. H. Kochem, W. Sohn, K. Jung, H. Ehrhardt, and E. S. Chang, J. Phys. B 18, 1253 (1985).

[12] K. D. Jordan and P. D. Burrow, Acc. Chem. Res. 11, 341 (1978).

[13] F. A. Gianturco and T. Stoecklin, J. Phys. B 27, 5903 (1994).

[14] J. Fedor, O. May, and M. Allan, Phys. Rev. A 78, 032701 (2008).

[15] T. A. Field, E. Slattery, D. J. Adams, and D. D. Morrison, J. Phys. B 38, 255 (2005).

[16] F. H. Read and N. J. Bowring, Charged Particle Optics Program CPO-3D, www.electronoptics.com

[17] O. J. Orient and S. K. Srivastava, Chem. Phys. Lett. 96, 681 (1983).

[18] Y. Itikawa, J. Phys. Chem. Ref. Data 31, 749 (2002).

[19] M. Knudsen, Ann. Phys. (Leipzig) 31, 205 (1910).

[20] M. Allan and S. F. Wong, Phys. Rev. Lett. 41, 1791 (1978).

[21] S. T. Chourou and A. E. Orel, Phys. Rev. A (to be published). 